\title{
Accurate Solutions of Initial Value Problems for Ordinary Differential Equations with the Fourth Order Runge Kutta Method
}

\author{
Md. Amirul Islam ${ }^{1}$ \\ ${ }^{1}$ School of Science and Engineering, Uttara University, Dhaka-1216, Bangladesh \\ Correspondence: Md. Amirul Islam, Department of Mathematics, Uttara University, Robiul Tower, Mirpur-1, \\ Dhaka-1216, Bangladesh. E-mail: amirul.math@gmail.com
}

\author{
Received: May 2, 2015 Accepted: May 16, 2015 Online Published: July 11, 2015 \\ doi:10.5539/ijsp.v4n3p41 URL: http://dx.doi.org/10.5539/ijsp.v4n3p41
}

\begin{abstract}
In this paper, we consider fourth order Runge-Kutta method for solving ordinary differential equations in initial value problems. The proposed methods are quite efficient and are practically well suited for solving these problems. Several examples are presented to demonstrate the accuracy and easy implementation of the proposed methods. The results of numerical experiments are compared with the analytical solution and thereby gain some insight into the accuracy of proposed methods. Finally we investigate and compute the error of proposed methods. This counterintuitive result is analyzed in this paper.
\end{abstract}

Keywords: initial value problem, runge-kutta method, error analysis

\section{Introduction}

Many problems of mathematical physics can be started in the form of differential equations. These equations also occur as reformulations of other mathematical problems such as ordinary differential equations and partial differential equations. Numerical methods are commonly used for solving mathematical problems that are formulated in science and engineering where it is difficult or even impossible to obtain exact solutions. Only a limited number of differential equations can be solved analytically. There are many analytical methods for finding the solution of ordinary differential equations. Even then there exist a large number of ordinary differential equations whose solutions cannot be obtained in closed form by using well known analytical methods, where we have to use the numerical methods to get the approximate solution of a differential equation under the prescribed initial condition or conditions. There are many types of practical numerical methods for solving initial value problems for ordinary differential equations.

From the literature review we may realize that several works in numerical solutions using fourth order Runge-Kutta method and many authors have attempted to solve initial value problems (IVP) to obtain high accuracy rapidly by using a numerous methods, such as Euler methods, and also some other methods. In this paper we apply fourth order Runge-Kutta method for solving ordinary differential equation in initial value problems. A more robust and intricate numerical technique is the Runge-Kutta method. This method is the most widely used one since it gives reliable starting values and is particularly suitable when the computation of higher derivatives is complicated. Finally two examples of different kinds of ordinary differential equations are given to verify the proposed formulation. The results of each numerical example indicate the convergence and error analysis is discussed to illustrate the efficiency of the method.

\section{Initial Value Problem}

In this paper we introduce a first-order differential equation is an Initial value problem (IVP) of the form,

$$
y^{\prime}(x)=f(x, y(x))
$$

with initial condition

$$
y\left(x_{0}\right)=y_{0}
$$

Numerical methods employ the differential equation (1) and the condition (2) to obtain approximations to the values of the solutions corresponding to various selected values of $x$. 


\section{Problem Formulation}

This method was devised by two German mathematicians, Runge about 1894 and extended by Kutta a few years later. The Runge -Kutta methods are designed to give greater accuracy and they possess the advantage of requiring only the function values at some selected points on the subinterval. The Runge -Kutta methods are distinguished by their orders in the sense that they agree with Taylor's series solution up to terms of $h^{r}$, where $\mathrm{r}$ is the order of the method. These methods do not demand prior computational of higher derivatives of $y(x)$ as in Taylor's series method. Fourth order Runge-Kutta methods are widely used for finding the numerical solutions of linear and non-linear ordinary differential equation. The general form of $4^{\text {th }}$ order Runge-Kutta method is

$$
\begin{aligned}
y_{n+1}(x)=y_{n}(x)+ & \frac{1}{6}\left(k_{1}+2 k_{2}+2 k_{3}+k_{4}\right), n=0,1,2,3, \ldots \\
\text { Where, } k_{1} & =h f(x, y), \\
k_{2} & =h f\left(x+\frac{h}{2}, y+\frac{k_{1}}{2}\right), \\
k_{3} & =h f\left(x+\frac{h}{2}, y+\frac{k_{2}}{2}\right), \\
\text { and } k_{4}= & h f\left(x+h, y+k_{3}\right) .
\end{aligned}
$$

\section{Error Analysis}

Errors in numerical solution of ordinary differential equations. There are two types of errors in numerical solutions. Round-off errors and Truncation errors occur when ordinary differential equations are solved numerically. Rounding errors originate from the fact that computers can only represent numbers using a fixed and limited number of significant figures. Thus, such numbers or cannot be represented exactly in computer memory. The discrepancy introduced by this limitation is call Round-off error. Truncation errors in numerical analysis arise when approximations are used to estimate some quantity. The accuracy of the solution will depend on how small we make the step size, h. A numerical method is said to be convergent if the numerical solution approaches the exact solution as the step size $h$ goes to 0 . In this paper we consider two IVP problems to verify accuracy of the proposed method. Then using this method we find numerical approximations for desired IVP. All the computations are performed by Mathematica software. The convergence of IVP is calculated by $e_{n}=\left|y\left(x_{n}\right)-y_{n}\right|<\delta$ where $y\left(x_{n}\right)$ denotes the approximate solution and $y_{n}$ denotes the exact solution and $\delta$ depends on the problem which varies from $10^{-7}$. The absolute error for this formula is defined by absolute error $=\left|y\left(x_{n}\right)-y_{n}\right|$.

\section{Numerical Examples}

Example 1: we consider the initial value problem $y^{\prime}(x)=x^{2}-y, y(0)=1$ on the interval $0 \leq x \leq 1$. The exact

\begin{tabular}{|c|c|c|c|c|}
\hline \multirow{2}{*}{$x_{n}$} & \multirow{2}{*}{ Exact solution } & \multicolumn{3}{|c|}{ Approximation } \\
\hline & & $h=0.1$ & $h=0.05$ & $h=0.0125$ \\
\hline 0.1 & 0.9051625819640405 & 0.9051627083333333 & 0.9051625897540301 & 0.9051625819941439 \\
\hline 0.2 & 0.8212692469220182 & 0.8212694954348958 & 0.8212692622282467 & 0.8212692469811308 \\
\hline 0.3 & 0.749181779318282 & 0.7491821454089058 & 0.7491818018485552 & 0.7491817794052449 \\
\hline 0.4 & 0.6896799539643608 & 0.6896804328297642 & 0.6896799834140293 & 0.6896799540779727 \\
\hline 0.5 & 0.6434693402873666 & 0.6434699269739351 & 0.6434693763443438 & 0.6434693404264032 \\
\hline 0.6 & 0.6111883639059736 & 0.6111890533816113 & 0.611188406254909 & 0.6111883640692017 \\
\hline 0.7 & 0.5934146962085904 & 0.593415483422517 & 0.5934147445343176 & 0.593414696394782 \\
\hline 0.8 & 0.5906710358827785 & 0.5906719158146551 & 0.5906710898731354 & 0.5906710360907194 \\
\hline 0.9 & 0.6034303402594008 & 0.6034313079592762 & 0.6034303996075129 & 0.6034303404879005 \\
\hline 1.0 & 0.6321205588285577 & 0.6321216094489349 & 0.6321206232346572 & 0.6321205590764547 \\
\hline
\end{tabular}
solution of the given problem is given by $y(x)=x^{2}-2 x-e^{-x}+2$.The results obtained are shown in Table 1(a) and Table 1(b) and the graphs of the numerical values of $y(x)$ with absolute error are displayed in figures 1, 1.1, 1.2 and 1.3 .

Table 1(a). Numerical Approximation for different step size. 
Table1 (b). Observed Maximum errors for example1.

\begin{tabular}{cccc}
\hline & & Errors & \\
\cline { 2 - 4 }$x_{n}$ & $h=0.1$ & $h=0.05$ & $h=0.0125$ \\
\hline 0.1 & $1.2636929280773757 \mathrm{e}-7$ & $7.789989542317244 \mathrm{e}-9$ & $3.010358629040866 \mathrm{e}-11$ \\
0.2 & $2.4851287760974117 \mathrm{e}-7$ & $1.53062285068728 \mathrm{e}-8$ & $5.911260370083937 \mathrm{e}-11$ \\
0.3 & $3.660906238156514 \mathrm{e}-7$ & $2.2530273202647777 \mathrm{e}-8$ & $8.696288134046881 \mathrm{e}-11$ \\
0.4 & $4.788654034415529 \mathrm{e}-7$ & $2.944966848161812 \mathrm{e}-8$ & $1.1361211971205876 \mathrm{e}-10$ \\
0.5 & $5.866865685488776 \mathrm{e}-7$ & $3.6056977181786465 \mathrm{e}-8$ & $1.3903689310978962 \mathrm{e}-10$ \\
0.6 & $6.894756376940592 \mathrm{e}-7$ & $4.234893535848272 \mathrm{e}-8$ & $1.6322809770485947 \mathrm{e}-10$ \\
0.7 & $7.872139266007494 \mathrm{e}-7$ & $4.832572719770667 \mathrm{e}-8$ & $1.8619150665699635 \mathrm{e}-10$ \\
0.8 & $8.799318765850828 \mathrm{e}-7$ & $5.399035696296295 \mathrm{e}-8$ & $2.0794088673170563 \mathrm{e}-10$ \\
0.9 & $9.676998754537536 \mathrm{e}-7$ & $5.934811186936173 \mathrm{e}-8$ & $2.2849988567941182 \mathrm{e}-10$ \\
1.0 & $10.506203772520095 \mathrm{e}-7$ & $6.440609956470666 \mathrm{e}-8$ & $2.4789748032105763 \mathrm{e}-10$ \\
\hline
\end{tabular}
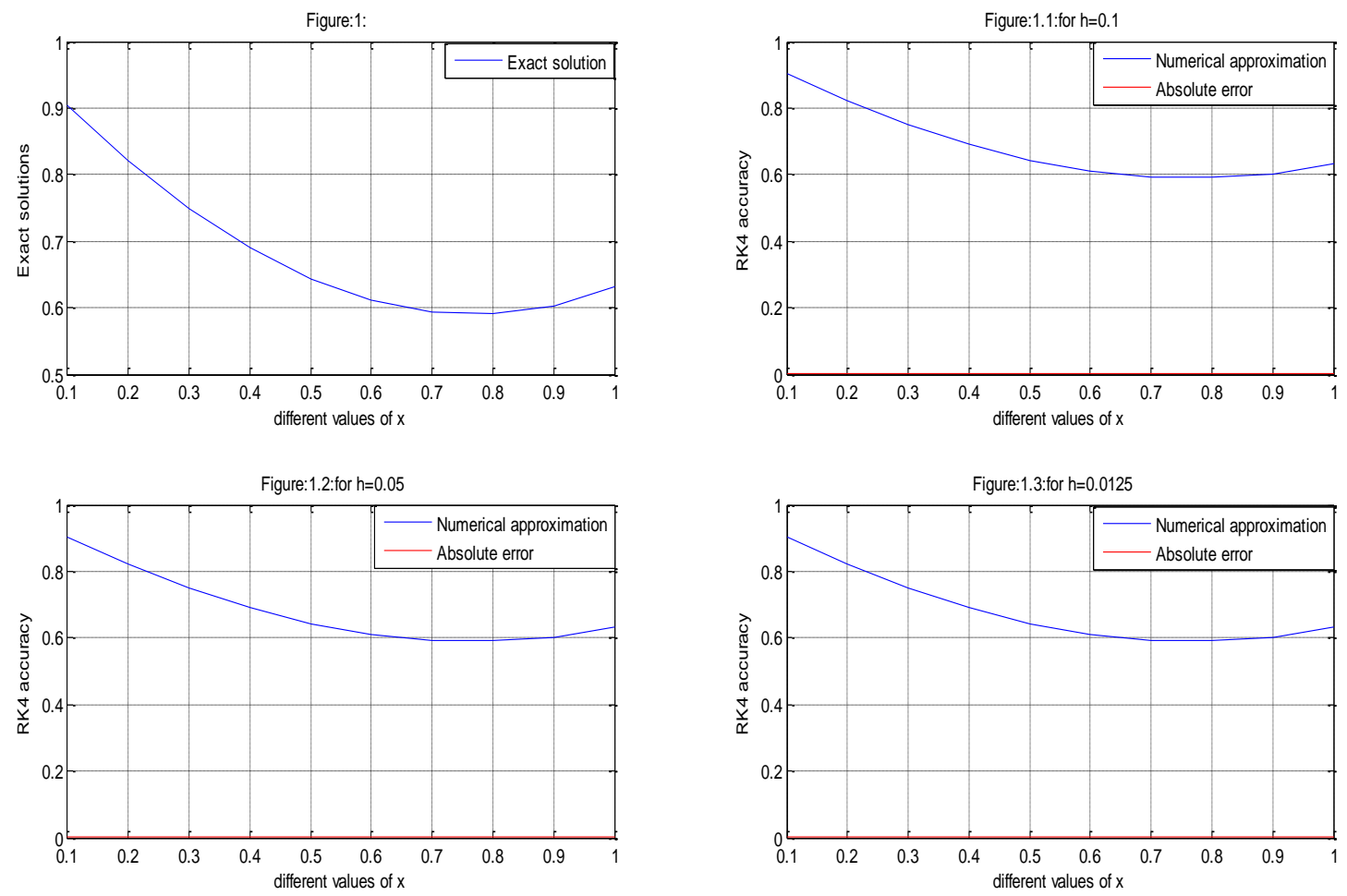

Example 2: we consider the initial value problem $y^{\prime}(x)=3 x+\frac{y}{2}, y(0)=1$ on the interval $0 \leq x \leq 1$. The exact solution of the given problem is given by $y(x)=13 e^{\frac{x}{2}}-6(x+2)$. The results obtained are shown in Table 2(a) and Table 2(b) and the graphs of the numerical values of $y(x)$ with absolute error are displayed in figures 2, 2.1, 2.2 and 2.3. 
Table 2(a). Numerical Approximation for different step size.

\begin{tabular}{ccccc}
\hline \multirow{2}{*}{$x_{n}$} & Exact solution & \multicolumn{3}{c}{ Approximation } \\
\cline { 3 - 5 } & & $h=0.1$ & $h=0.05$ & $h=0.0125$ \\
\hline 0.1 & 1.0665242528883123 & 1.0665242187500000 & 1.0665242507097914 & 1.0665242528796686 \\
0.2 & 1.1672219349834183 & 1.1672218632061766 & 1.1672219304029865 & 1.1672219349652448 \\
0.3 & 1.3038451554676804 & 1.3038450422816700 & 1.303845148244766 & 1.3038451554390216 \\
0.4 & 1.4782358560822093 & 1.4782356974299664 & 1.478235845957886 & 1.478235856042037 \\
0.5 & 1.6923304169406386 & 1.6923302084574950 & 1.6923304036363807 & 1.692330416887851 \\
0.6 & 1.9481644984880404 & 1.9481642354812763 & 1.9481644817043813 & 1.9481644984214466 \\
0.7 & 2.2478781317123406 & 2.2478778091390340 & 2.2478781111274726 & 2.247878131630668 \\
0.8 & 2.5937210693365174 & 2.5937206817799456 & 2.593721044604765 & 2.593721069238384 \\
0.9 & 2.9880584113721937 & 2.9880579530167990 & 2.9880583821224502 & 2.9880584112561377 \\
1.0 & 3.433376519101664 & 3.4333759837063567 & 3.433376484935653 & 3.433376518966102 \\
\hline
\end{tabular}

Table2 (b). Observed Maximum errors for example2.

\begin{tabular}{cccc}
\hline & \multicolumn{2}{c}{ Errors } \\
\cline { 2 - 4 } & $h=0.1$ & $h=0.05$ & $h=0.0125$ \\
\hline 0.1 & $3.413831239917897 \mathrm{e}-8$ & $2.1785209192159982 \mathrm{e}-9$ & $8.643752380521619 \mathrm{e}-12$ \\
0.2 & $7.177724170581712 \mathrm{e}-8$ & $4.5804318116182685 \mathrm{e}-9$ & $1.8173462734694112 \mathrm{e}-11$ \\
0.3 & $1.131860103420479 \mathrm{e}-7$ & $7.22291448873591 \mathrm{e}-9$ & $2.865707671162454 \mathrm{e}-11$ \\
0.4 & $1.5865224289868252 \mathrm{e}-7$ & $1.012432337965663 \mathrm{e}-8$ & $4.0168757209357864 \mathrm{e}-11$ \\
0.5 & $2.084831436377499 \mathrm{e}-7$ & $1.3304257917567952 \mathrm{e}-8$ & $5.2791104820926193 \mathrm{e}-11$ \\
0.6 & $2.630067641451461 \mathrm{e}-7$ & $1.6783659129160355 \mathrm{e}-8$ & $6.659384155227599 \mathrm{e}-11$ \\
0.7 & $3.225733067324654 \mathrm{e}-7$ & $2.0584868032358372 \mathrm{e}-8$ & $8.167599929720382 \mathrm{e}-11$ \\
0.8 & $3.8755657172373503 \mathrm{e}-7$ & $2.4731748649031715 \mathrm{e}-8$ & $9.812994861135849 \mathrm{e}-11$ \\
0.9 & $4.5835539452809826 \mathrm{e}-7$ & $2.924974351614651 \mathrm{e}-8$ & $1.1605250094248731 \mathrm{e}-10$ \\
1.0 & $5.353953071995932 \mathrm{e}-7$ & $3.4166014462044814 \mathrm{e}-8$ & $1.3555823130673161 \mathrm{e}-10$ \\
\hline
\end{tabular}

\section{Discussion of Results}

The calculated results are displayed in Table 1(a), 1(b) and Table 2(a), 2(b) and graphically represent in Figures(1)-(1.3) and Figures (2)-(2.3). Reasonably good results are obtained even for a moderately large step size and the approximation can be improved by decreasing the step size. The proposed methods give very good result when compared with the exact solution. In principle, we say that a numerical solution converges to the exact solution if decreasing the step size leads to decrease errors such that in the limit when the step size to zero the errors go to zero.

\section{Conclusion}

In this paper, we have used fourth order Runge- Kutta methods for solving ordinary differential equation in initial value problems (IVP). To achieve the desired accuracy of numerical solution it is necessary to take step size very, very small. Therefore, it is an important task to make a proper balance between accuracy and efficiency of the solutions. The numerical results obtained by the proposed method are in good agreement with the exact solutions. The results of each problem guarantee the convergence and stability. We may notice that the accuracy increase with decrease step size, we may realize that this method may be applied to solve IVP to find the desired accuracy. 

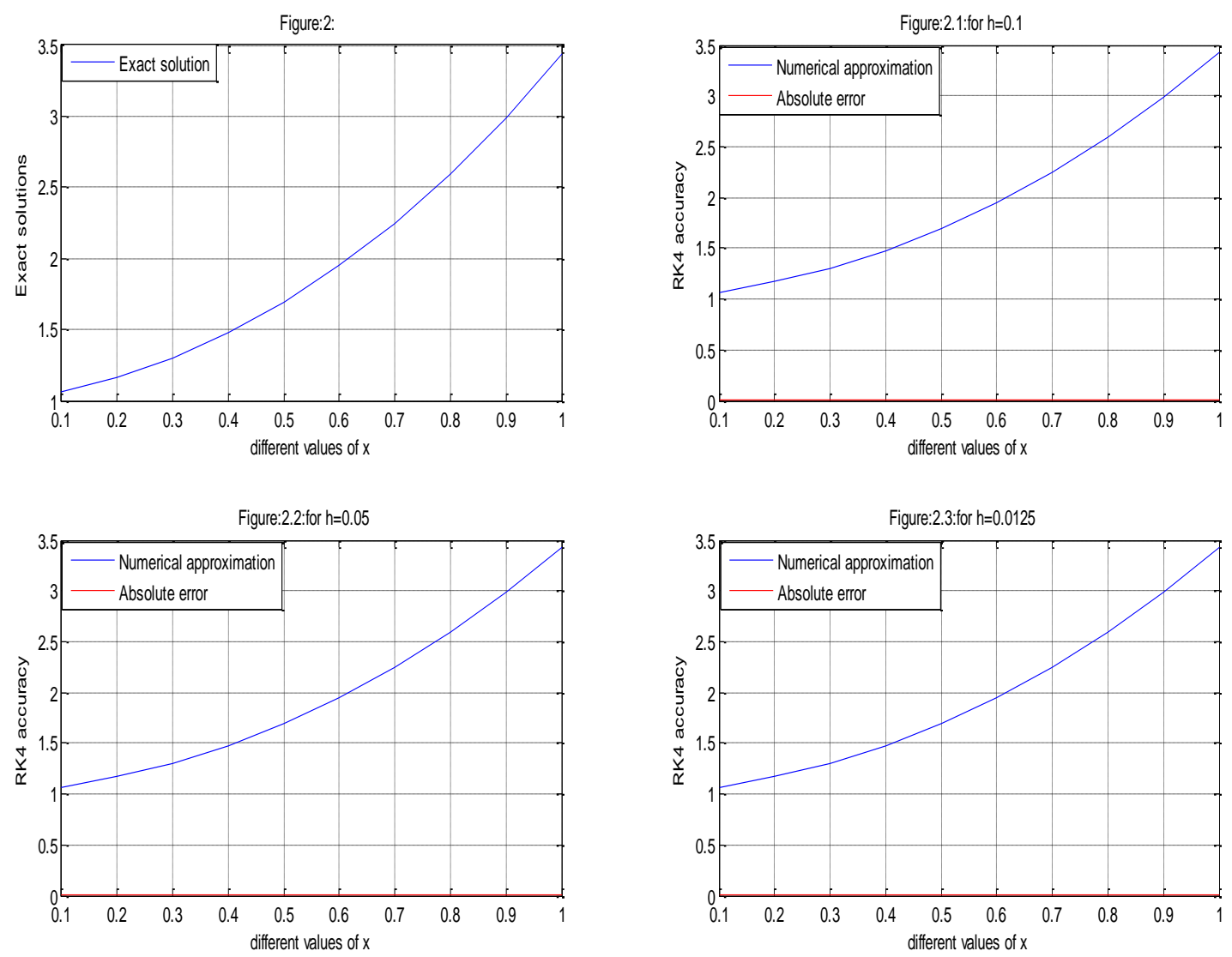

\section{References}

Balagurusamy, E. (2006). Numerical Methods, Tata McGraw - Hill. New Delhi, India.

Eaqub Ali, S. M. (2010). A Text Book of Numerical Methods with Computer programming. Beauty publication, Khulna-9100, Bangladesh.

Gear, C. W. (1971). Numerical Initial value problems in ordinary differential equations, Prentice- Hall.

Hall, G., \& Watt, J. M. (1976). Modern Numerical methods for ordinary differential equations, Oxford University Press.

Hossain, M. S., Bhattacharjee, P. K., \& Hossain, M. E. (2013). Numerical Analysis, Titas Publications, Dhaka.

Kockler, N. (1994). Numerical Method for Ordinary Systems of Initial value Problems. John Wiley and Sons: New York, Ny.

Lambert, J. D. (1973). Computational Methods in ordinary differential equations, Wiley.

Ogunrinde, R. B., Fadugba, S. E., \& Okunlola, J. T. (2012). On Some Numerical Methods for Solving Initial value Problems in ordinary Differential equations. IOSR Journal of Mathematics, 1(3), $25-31$. http://dx.doi.org/10.9790/5728-0132531

Sastry, S. S. (2008). Introductory Methods of numerical Analysis. Prentice-Hall, India.

Shampine, L. F., \& Watts, H. A. (1971). Comparing Error Estimators for Runge-Kutta methods. Mathematics of computation, 25(115), 445-455. http://dx.doi.org/10.1090/S0025-5718-1971-0297138-9

\section{Copyrights}

Copyright for this article is retained by the author(s), with first publication rights granted to the journal.

This is an open-access article distributed under the terms and conditions of the Creative Commons Attribution license (http://creativecommons.org/licenses/by/3.0/). 